\title{
Watching dark matter stars burn - possible signatures of Dark Stars in the EBL
}

\author{
Andreas Maurer ${ }^{*, a}$, Martin Raue ${ }^{a}$, Tanja Kneiske ${ }^{a}$, Dominik Elsässer ${ }^{b}$, Peter H. \\ Hauschildt ${ }^{c}$, and Dieter Horns ${ }^{a}$ \\ ${ }^{a}$ Institut für Experimentalphysik, Universität Hamburg \\ Luruper Chaussee 149, D-22761 Hamburg, Germany \\ ${ }^{b}$ Institut für Theoretische Physik und Astrophysik \\ Am Hubland, D-97074 Würzburg, Germany \\ ${ }^{c}$ Hamburger Sternwarte \\ Gojenbergsweg 112, D-21029 Hamburg, Germany \\ E-mail: andreas.maurerephysik.uni-hamburg.de, \\ martin.raueephysik.uni-hamburg.de, \\ tanja.kneiske@physik.uni-hamburg.de, \\ elsaesserdastro.uni-wuerzburg.de, yeti@hs.uni-hamburg.de, \\ dieter.hornsephysik.uni-hamburg.de
}

\begin{abstract}
Recently, it has been proposed that self-annihilating dark matter could have a significant effect on the formation and development of the first stars in the universe. In such a model, the energy released by the self-annihilating dark matter may be the main power source for this class of young stellar objects called Dark Stars (DS). Their features (e.g. luminosity, temperature, lifetime) could differ from normal PopIII stars and therefore makes them distinguishable. The contribution of DS to the extragalactic background light (EBL) considering multiple initial parameters is calculated. By comparing our results with existing EBL data and limits we can derive first observational constraints on Dark Stars in the early universe. Future observations (e.g. with JWST) will improve these results.
\end{abstract}

Identification of Dark Matter 2010

July 26 - 302010

University of Montpellier 2, Montpellier, France

\footnotetext{
* Speaker.
} 


\section{Introduction}

Astrophysical observations from the last decades hint towards a large mass contribution in the universe that is of a unknown, dark nature. Large surveys (e.g. SDSS [1] , 2dF [2] ) and numerical simulations like the Millennium run [3] point towards a convincing scenario of large scale structure formation within a cold dark matter (DM) model in an accelerated expanding universe ( $\Lambda \mathrm{CDM})$. A good particle candidate for the DM content are self-annihilating WIMPs (Weakly Interacting Massive Particle) that could effect the physics of the first stars (see e.g. [4, 5] for a review article).

The epoch of the first star formation in the universe is not yet observable with today's astronomical instruments. The circumstances and mechanisms of these processes are still topics of ongoing analysis and rely on sophisticated numerical simulations (for a review, see e.g. [6]). In the last two years several studies discussed the role of WIMP dark matter on the formation of the first stars [7, 8]. Assuming that self-annihilating particles provide the dark matter content of the universe, it is believed that this new source of energy injection into the first stars may alter their features remarkably. The energy injection of these self-annihilating WIMPs delay or even prevent the nuclear hydrogen burning which is normally the main energy source of stars. Two mechanisms are available to supply the star with high DM densities: adiabatic contracted DM due to the gravitational pull from the baryons that form the star (as investigated by [7]) and elastic scattering between WIMPs and baryons (see e.g. [8]). There can also be the possibility for a combination of these two mechanisms with different relative efficiencies. The exact physical process that provides the dark matter powered stars (Dark Star; DS) with "fuel" has no great impact on this work as long as there is a high enough dark matter density in the center of the DS. In both cases the resulting features of these stellar objects can be quite similar [8,9]. After the "dark phase" the star is thought to evolve as a normal zero age main sequence (ZAMS) star according to its mass.

Instead of considering the very challenging direct detection of DS (see e.g. [10]), the approach here is to search for signatures of DS in the diffuse metagalactic radiation field (MRF). The local optical to infrared part of the MRF is also known as extragalactic background light (EBL, for a review see e.g. [11]). Its main contribution originates from integrated starlight and thermal dust emissions of all cosmic epochs. This fact makes the EBL an ideal probe for the star formation history of the universe and therefor offers a unique possibility to search for emission in the early universe, e.g. investigated in [12]. There are different types of observational strategies for the EBL. Lower limits to the EBL are derived from galaxy number counts which are available up to a redshift $\approx 2$ from Hubble [13] and Spitzer [14]. Upper limits can be derived from direct observations (COBE [15]), but these are presumably polluted by prominent foreground emission. A different method for obtaining upper limits on the EBL density makes use of the spectra from very high energy (VHE) $\gamma$-ray sources, in particular blazars (see e.g. [16]). These limits deliver the possibility to constrain DS scenarios.

For all calculations here a flat Friedman cosmology is adopted with $\Omega_{m}=0.3, \Omega_{\Lambda}=0.7$ and a Hubble constant of $H_{0}=70 \mathrm{~km} \mathrm{~s}^{-1} \mathrm{Mpc}^{-1}$.

\section{Method}

To calculate the contribution of dark matter powered stars to the extragalactic background light 


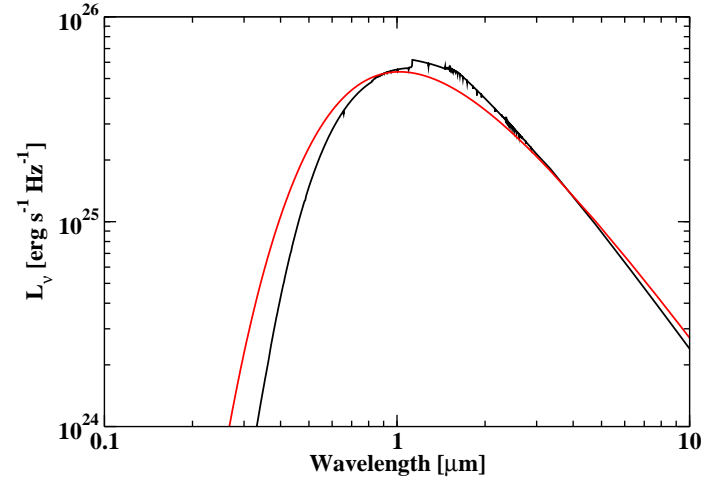

(a) Specific luminosity for a modelled DS atmosphere (black) and a blackbody (red) with same temperature and radius. DS parameters are $R_{\mathrm{DS}}=2.4 \times 10^{12} \mathrm{~m}$, $M_{\mathrm{DS}}=106 \mathrm{M}_{\odot}, T_{\mathrm{DS}}=5000 \mathrm{~K}$

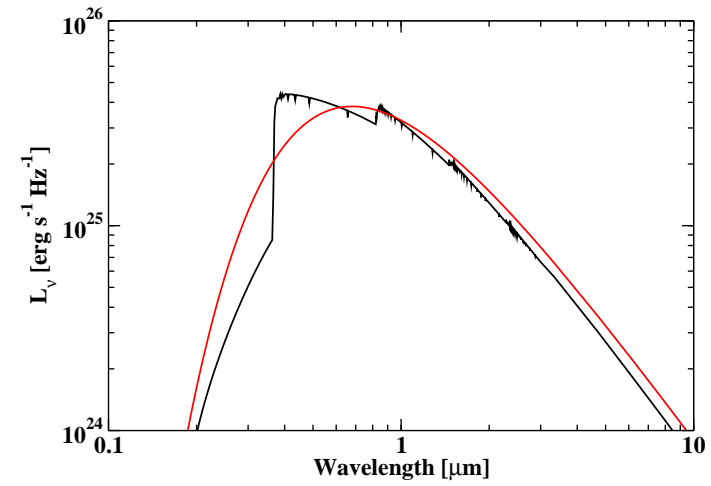

(b) Same as in (a). DS parameters are $R_{\mathrm{DS}}=1.1 \times$ $10^{12} \mathrm{~m}, M_{\mathrm{DS}}=690 \mathrm{M}_{\odot}, T_{\mathrm{DS}}=7500 \mathrm{~K}$

Figure 1: Model spectra used as input parameters. DS features taken from [9].

a forward evolution model is used, similar to [17]. In the following the individual input parameters of the model are described.

The spectra of DS are calculated with the model atmosphere package PHOENIX, version 16 [18]. For the model atmospheres used here the abundance of $\mathrm{H}$ was set to 0.92 by number (mass fraction: 0.75 ) and that of He was set to 0.78 by number (mass fraction: 0.25 ) for all models, all other elements (including $\mathrm{Li}$ ) have an abundance of zero in these models. DS spectra with effective temperatures $5000 \mathrm{~K}$ and $7500 \mathrm{~K}$ and with parameters taken from [9] have been computed (see Fig. 1).

Independent of the exact mechanism powering the DM burning, models predict a stable phase which dominates the total radiative output of the DS [7, 8]. During this phase the luminosity is nearly constant (see e.g. Figure 2 in [9], Figure 4 in [8] and Figure 1 in [19]). The exact length of the DS lifetime $\left(\Delta t_{\mathrm{DS}}\right)$ is highly uncertain and depends on various factors, e.g. DM type, DS model, DM halo profile, etc (for an extensive discussion see [10]). In this work a wide band of possible DS lifetimes is explored ranging from $10^{5}-10^{9}$ years.

A crucial parameter, to which the EBL flux is sensitive, is the formation density of DS. This quantity is directly linked to the cosmic star formation rate (SFR) $\dot{\rho}_{*}(z)$ for the first stars (PopIII) which can be expressed as a comoving mass formation rate in units of $\mathrm{M}_{\odot}$ year $^{-1} \mathrm{Mpc}^{-3}$. A constant SFR for Dark Stars is adopted between the maximum $\left(z_{\max }\right)$ and the minimum $\left(z_{\min }\right)$ redshift of their formation epoch. The linear scaling factor $\mathrm{SFR}_{\text {Norm }}$ ranges from $10^{-7}$ to $10^{-3}$ comparable to typical values obtained for the first stars [20].

The influence of $z_{\max }$ on the resulting EBL turns out to be negligible and so in the following its value is fixed at 30 . The investigated range of $z_{\min }$ reaches from 5 to 15 according to published PopIII formation periods [21, 22].

\section{Results \& Conclusion}

The resulting EBL contribution is calculated for different sets of parameters. Both DS spectra 


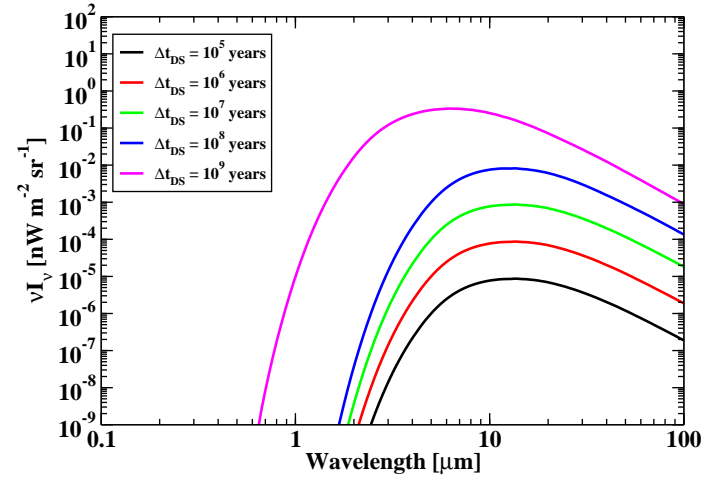

(a) EBL contribution for different DS lifetimes. DS parameters are $R_{\mathrm{DS}}=2.4 \times 10^{12} \mathrm{~m}, M_{\mathrm{DS}}=106 \mathrm{M}_{\odot}, T_{\mathrm{DS}}$ $=5000 \mathrm{~K}$

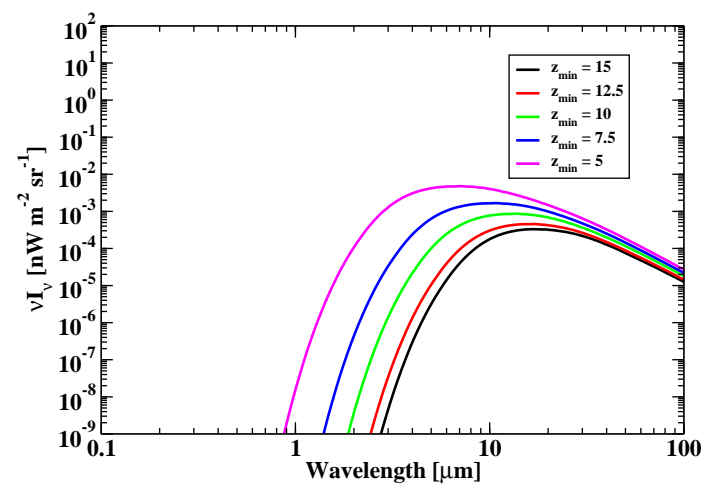

(c) EBL contribution for different values of $z_{\min }$. DS parameters are $R_{\mathrm{DS}}=2.4 \times 10^{12} \mathrm{~m}, M_{\mathrm{DS}}=106 \mathrm{M}_{\odot}, T_{\mathrm{DS}}$ $=5000 \mathrm{~K}$

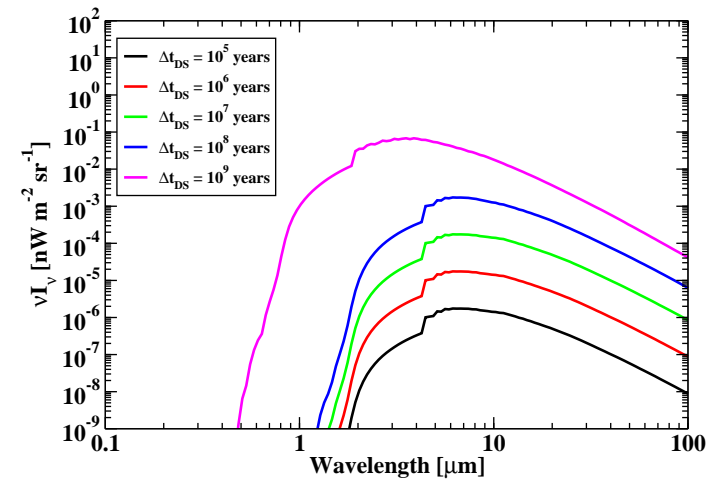

(b) Same as in (a). DS parameters are $R_{\mathrm{DS}}=1.1 \times$ $10^{12} \mathrm{~m}, M_{\mathrm{DS}}=690 \mathrm{M}_{\odot}, T_{\mathrm{DS}}=7500 \mathrm{~K}$

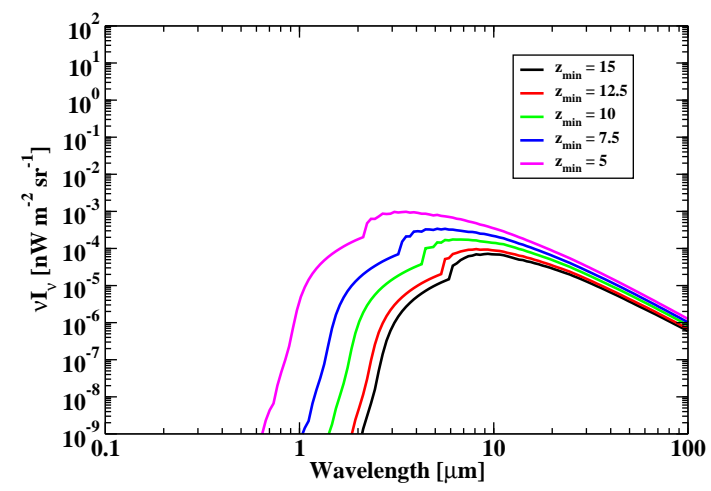

(d) Same as in (c). DS parameters are $R_{\mathrm{DS}}=1.1 \times$ $10^{12} \mathrm{~m}, M_{\mathrm{DS}}=690 \mathrm{M}_{\odot}, T_{\mathrm{DS}}=7500 \mathrm{~K}$

Figure 2: EBL signatures for two DS models. The resulting EBL fluxes for different DS lifetimes $\left(\Delta t_{\mathrm{DS}}\right)$ and minimum redshifts of the DS formation epoch $\left(z_{\min }\right)$ are shown.

\begin{tabular}{cccc}
\hline \hline & $\Delta t_{\mathrm{DS}}$ & $z_{\min }$ & $\mathrm{SFR}_{\text {Norm }}$ \\
\hline $\min$ & $10^{5}$ & 5 & $10^{-7}$ \\
fiducial & $10^{7}$ & 10 & $10^{-5}$ \\
$\max$ & $10^{9}$ & 15 & $10^{-3}$ \\
\hline
\end{tabular}

Table 1: Dark Star parameter range

presented in Fig. 1 are taken as model input and a wide range of possible EBL signatures are calculated. This is shown in Fig. 2; while varying one parameter in its allowed range, the others are set to a fiducial value. The details of the parameters are presented in table 1.

As SFR $_{\text {Norm }}$ merely acts as a linear scaling factor, no plots for different values of it are shown. By varying the end of the DS formation period two effects on the EBL flux can be observed (Fig. 2(c) and Fig. 2(d)). Decreasing $z_{\min }$ leads to a longer DS formation time span und thus the total amount of emitted photons is increased and with it the total contribution to the EBL. The second consequence is a wavelength dependency for the peak value of the EBL signature. This is caused by a non-zero value of the comoving luminosity density (emissivity) at lower redshift that 
leads to less redshifted peak wavelengths.

The influence of different DS lifetimes is displayed in Fig. 2(a) and Fig. 2(b). For DS lifetimes smaller than the formation period $t\left(z_{\min }\right)-t\left(z_{\max }\right)$ the resulting EBL scales linearly with increasing $\Delta t_{\mathrm{DS}}$. At higher lifetimes than $\sim 10^{8}$ years the intensity of the EBL is increased to a greater amount as well as the peak value of the DS signature is shifted towards lower wavelengths. This is caused by a non-vanishing emissivity at redshifts $z<z_{\min }$. If $\Delta t_{\mathrm{DS}}$ is short enough, the end of DS formation is roughly equal to the time when DS cease to emit photons as the amount of DS drops off almost instantly. When considering DS lifetimes higher than $\sim 10^{8}$ years the declining population of DS causes a residual emissivity at $z<z_{\min }$ and explains the shifted peak of the EBL signature as well as the higher contribution to the total EBL flux (see in Fig. 2(a) and in Fig. 2(b) the results for $\Delta t_{\mathrm{DS}}=10^{9}$ years).

Considering the chosen parameter ranges (cf. table 1), peak EBL contributions from $10^{-9}$ to roughly $70 \mathrm{nW} \mathrm{m}{ }^{-2} \mathrm{sr}^{-1}$ can be obtained. As Fig. 2 shows, the locations of the maximum EBL flux are located roughly between $1-10 \mu \mathrm{m}$. In this wavelength regime the EBL flux is measured between $\sim 10$ (lower limits) and $\sim 100 \mathrm{nW} \mathrm{m}^{-2} \mathrm{sr}^{-1}$ (upper limits) ${ }^{1}$. This shows that EBL contributions from DS can reach into the detectable range of the EBL flux and thus can deliver constraints for DS parameters. Further work on the constraints delivered by EBL contributions will be published in [23]. Future efforts to measure the EBL via direct observations (e.g. with the James Webb Space Telescope) and to derive refined upper limits (e.g. measurements of VHE $\gamma$-ray sources with forthcoming Cherenkov telescopes like CTA) will improve the possibility to put constraints on Dark Stars enormously.

\section{Acknowledgments}

The participation in the 8th International Workshop of Identification of Dark Matter was made possible with the support of the Cluster of Excellence: Connecting Particles with the Cosmos of the Landesexzellenzinitiative Hamburg. This work has made use of NASA's Astrophysics Data System Service.

\section{References}

[1] K. N. Abazajian, J. K. Adelman-McCarthy, M. A. Agüeros, S. S. Allam, C. Allende Prieto, D. An, K. S. J. Anderson, S. F. Anderson, J. Annis, N. A. Bahcall, and et al., The Seventh Data Release of the Sloan Digital Sky Survey, Astrophys. J., Suppl. Ser. 182 (June, 2009) 543-558, [0 812.0649 ].

[2] M. Colless, B. A. Peterson, C. Jackson, J. A. Peacock, S. Cole, P. Norberg, I. K. Baldry, C. M. Baugh, J. Bland-Hawthorn, T. Bridges, R. Cannon, C. Collins, W. Couch, N. Cross, G. Dalton, R. De Propris, S. P. Driver, G. Efstathiou, R. S. Ellis, C. S. Frenk, K. Glazebrook, O. Lahav, I. Lewis, S. Lumsden, S. Maddox, D. Madgwick, W. Sutherland, and K. Taylor, The 2dF Galaxy Redshift Survey: Final Data Release, ArXiv Astrophysics e-prints (June, 2003) [astro-ph/0306581].

[3] V. Springel, S. D. M. White, A. Jenkins, C. S. Frenk, N. Yoshida, L. Gao, J. Navarro, R. Thacker, D. Croton, J. Helly, J. A. Peacock, S. Cole, P. Thomas, H. Couchman, A. Evrard, J. Colberg, and F. Pearce, Simulations of the formation, evolution and clustering of galaxies and quasars, Nature $\mathbf{4 3 5}$ (June, 2005) 629-636, [astro-ph/ 0504097 ].

\footnotetext{
${ }^{1}$ see e.g. [11] and references therein
} 
[4] G. Jungman, M. Kamionkowski, and K. Griest, Supersymmetric dark matter, Phys. Rep. 267 (Mar., 1996) 195-373, [hep-ph/9506380].

[5] G. Bertone, D. Hooper, and J. Silk, Particle dark matter: evidence, candidates and constraints, Phys. Rep. 405 (Jan., 2005) 279-390, [hep-ph/ 0404175 ].

[6] V. Bromm and R. B. Larson, The First Stars, Ann. Rev. Astron. Astrophys. 42 (Sept., 2004) 79-118, [astro-ph/0311019].

[7] D. Spolyar, K. Freese, and P. Gondolo, Dark Matter and the First Stars: A New Phase of Stellar Evolution, Physical Review Letters 100 (Feb., 2008) 051101-+, [astro-ph/0 05.0521 ].

[8] F. Iocco, A. Bressan, E. Ripamonti, R. Schneider, A. Ferrara, and P. Marigo, Dark matter annihilation effects on the first stars, Mon. Not. R. Astron. Soc. 390 (Nov., 2008) 1655-1669, [0 805.4016 ].

[9] D. Spolyar, P. Bodenheimer, K. Freese, and P. Gondolo, Dark Stars: A New Look at the First Stars in the Universe, Astrophys. J. 705 (Nov., 2009) 1031-1042, [0903.3070].

[10] E. Zackrisson, P. Scott, C. Rydberg, F. Iocco, B. Edvardsson, G. Östlin, S. Sivertsson, A. Zitrin, T. Broadhurst, and P. Gondolo, Finding High-redshift Dark Stars with the James Webb Space Telescope, Astrophys. J. 717 (July, 2010) 257-267, [1002.3368].

[11] M. G. Hauser and E. Dwek, The Cosmic Infrared Background: Measurements and Implications, Ann. Rev. Astron. Astrophys. 39 (2001) 249-307, [astro-ph/ 0105539 ].

[12] M. Raue, T. Kneiske, and D. Mazin, First stars and the extragalactic background light: how recent $\gamma$-ray observations constrain the early universe, Astron. Astrophys. 498 (Apr., 2009) 25-35, [0806.2574].

[13] P. Madau and L. Pozzetti, Deep galaxy counts, extragalactic background light and the stellar baryon budget, Mon. Not. R. Astron. Soc. 312 (Feb., 2000) L9-L15, [astro-ph/9907315].

[14] G. G. Fazio, M. L. N. Ashby, P. Barmby, J. L. Hora, J.-S. Huang, M. A. Pahre, Z. Wang, S. P. Willner, R. G. Arendt, S. H. Moseley, M. Brodwin, P. Eisenhardt, D. Stern, E. V. Tollestrup, and E. L. Wright, Number Counts at $3 \mu \mathrm{m}<\lambda<10 \mu \mathrm{m}$ from the Spitzer Space Telescope, Astrophys. J., Suppl. Ser. 154 (Sept., 2004) 39-43, [astro-ph/ 0405595 ].

[15] M. G. Hauser, R. G. Arendt, T. Kelsall, E. Dwek, N. Odegard, J. L. Weiland, H. T. Freudenreich, W. T. Reach, R. F. Silverberg, S. H. Moseley, Y. C. Pei, P. Lubin, J. C. Mather, R. A. Shafer, G. F. Smoot, R. Weiss, D. T. Wilkinson, and E. L. Wright, The COBE Diffuse Infrared Background Experiment Search for the Cosmic Infrared Background. I. Limits and Detections, Astrophys. J. 508 (Nov., 1998) 25-43, [astro-ph/9806167].

[16] D. Mazin and M. Raue, New limits on the density of the extragalactic background light in the optical to the far infrared from the spectra of all known TeV blazars, Astron. Astrophys. 471 (Aug., 2007) 439-452, [astro-ph/0701694].

[17] T. M. Kneiske, K. Mannheim, and D. H. Hartmann, Implications of cosmological gamma-ray absorption. I. Evolution of the metagalactic radiation field, Astron. Astrophys. 386 (Apr., 2002) 1-11, [astro-ph/0202104].

[18] P. H. Hauschildt and E. Baron, A 3D radiative transfer framework. VI. PHOENIX/3D example applications, Astron. Astrophys. 509 (Jan., 2010) A36+, [0911.3285].

[19] S. Yoon, F. Iocco, and S. Akiyama, Evolution of the First Stars with Dark Matter Burning, Astrophys. J., Lett. 688 (Nov., 2008) L1-L4, [0 806 . 2662]. 
[20] M. Trenti and M. Stiavelli, Formation Rates of Population III Stars and Chemical Enrichment of Halos during the Reionization Era, Astrophys. J. 694 (Apr., 2009) 879-892, [0901.0711].

[21] M. Trenti, M. Stiavelli, and J. Michael Shull, Metal-free Gas Supply at the Edge of Reionization: Late-epoch Population III Star Formation, Astrophys. J. 700 (Aug., 2009) 1672-1679, [0 905 . 4504 ].

[22] U. Maio, B. Ciardi, K. Dolag, L. Tornatore, and S. Khochfar, The transition from population III to population II-I star formation, Mon. Not. R. Astron. Soc. (June, 2010) 905-+, [1 003.4992$].$

[23] A. Maurer, et al. in preparation, . 